\title{
Too soon to translate?
}

\author{
An association between a retrovirus and chronic fatigue syndrome has courted controversy since it was first \\ announced. In light of new data discounting this link, medical decisions made on its basis-some of which were \\ encouraged by the patient advocate community—might have been premature.
}

n 2006, the gammaretrovirus xenotropic murine leukemia virusrelated virus (XMRV) was detected in human prostate tumor tissues (PLoS Pathog. 2, e25, 2006). Judy Mikovits and her colleagues from the Whittemore-Peterson Institute (WPI) then reported that this virus was also associated with chronic fatigue syndrome (CFS) (Science 326, 585-589, 2009). They found XMRV DNA in two thirds of the blood samples from individuals with CFS that they examined, as well as evidence of XMRV infectivity and patient immune responses to a closely related virus, spleen focus-forming virus. The WPI findings were debated as a flurry of subsequent reports failed to reproduce the results. In response, the US National Institutes of Health (NIH) has initiated a multicenter, blinded, large-scale study to compare blood samples from individuals with CFS to those from healthy subjects, with the results expected later this year.

Now, two new papers indicate that XMRV was a contaminant in the initial WPI study. The first of the studies could not find any evidence of XMRV infection in the same samples tested by the WPI (Science doi:10.1126/science.1204963, 2011). The authors also found that XMRV and related gammaretroviruses are inactivated by sera from people with CFS, indicating that these viruses are unlikely to establish an infection in humans. An independent study showed that XMRV probably arose as the consequence of a recombination event between two mouse proviruses during the passaging of human prostate tumor xenografts through mice (Science doi:10.1126/science.1205292, 2011). This recombination event occurred between 1993 and 1996, long after the first diagnosis of CFS.

When the WPI article was published, many scientists advised that further investigation would be necessary before moving forward to translate the findings. Others were not so cautious: a WPI-owned company now offers a test (costing up to \$549) to detect XMRV DNA in people with CFS, although it is unclear what benefit such knowledge would provide. Despite undesirable side effects and a lack of evidence of clinical benefit, some doctors have prescribed antiretroviral drugs to patients with CFS on the basis of the observation that antiretrovirals can inhibit XMRV replication in vitro (PLoS ONE, 5, e9948, 2010). Some blood banks have prohibited people with CFS from donating blood in response to an advisory from the AABB (formerly the American Association of Blood Banks), an international association of blood banks, and an advisory committee recently recommended that the US Food and Drug Administration should follow suit. All of these measures now seem premature, given the questionable strength of the data supporting them.

The debate over XMRV's association with CFS has also reached a fever pitch among people with CFS. There is currently no accurate diagnosis for CFS; instead, it is clinically defined by what it is not. Without a clear definition of the illness and little funding or attention being devoted to a better understanding of the condition, individuals with CFS have felt marginalized by the medical and scientific communities. Given that the WPI report pointed to potential therapeutic avenues for CFS, many affected individuals lent support to these findings and lobbied for them to be taken forward to influence clinical practice.

A key question is whether the WPI findings and their implications were properly understood by the patient advocate community. The authors never claimed that XMRV causes CFS; they reported that there was a 'highly significant association' between the two. Even if the ongoing NIH study confirms an association, it would not prove a cause and consequence relationship. For example, XMRV could be an opportunistic infection that takes advantage of the lowered immune defenses associated with fatigue, or it could be a co-infecting pathogen with the true causative infectious agent.

Could the eagerness of CFS sufferers to see their condition taken seriously have encouraged premature translation of the XMRV association? The influence of a passionate advocate community should not be underestimated. The multiple-sclerosis community recently lobbied the Canadian Institutes of Health Research into funding further research into venoplasty as a potential treatment for the condition, despite many experts advising against it owing to lack of clinical benefit and the inherent riskiness of the procedure (Nature, 472, 410-411, 2011). Illustrating the positive results of advocacy, Duchenne muscular dystrophy advocacy groups were essential for driving forward and funding a new therapeutic, developed by the Netherlands-based company Prosensa. This therapeutic, PRO051, has now been licensed to GlaxoSmithKline and recently entered phase 3 trials (Nat. Biotech. 28, 1145-1148, 2010).

Patient advocacy can drive the development of new therapies, but it is also essential to consider how scientists and clinicians can communicate with patients to best meet their needs. For example, some people with CFS fear that discrediting of the XMRV connection could adversely affect CFS research. They now need reassurance that other avenues of research will be explored and that their disease is still taken seriously. In this regard, more initiatives such as the UK-based James Lind Alliance, which works with individuals suffering from many different conditions and brings them together with clinicians to identify and prioritize potential treatments, generating information to feed back to funding bodies, could be helpful for opening the channels of communication.

The XMRV and CFS experience has demonstrated that the pressure to find causes and cures for diseases should not overrule the need for scientific proof and validation. There is a delicate balance between being too cautious and delivering medical care to those who urgently need it, but hopefully scientists, clinicians and patients can learn from this example and work together to ensure that the right scientific findings are translated at the appropriate time: once there is sufficient evidence to warrant moving forward. 